\title{
An R package for SNP marker-based parent-offspring tests
}

\author{
Hussein Abdel-Haleem ${ }^{1}$, Pengsheng $\mathrm{Ji}^{2}, \mathrm{H}$ Roger Boerma ${ }^{1,3}$ and Zenglu $\mathrm{Li}^{{ }^{*}}$
}

\begin{abstract}
Background: With the advancement of genotyping technologies, whole genome and high-density SNP markers have been widely used for genotyping of mapping populations and for characterization of germplasm lines in many crops. Before conducting SNP data analysis, it is necessary to check the individuals to ensure the integrity of lines for further data analysis.

Results: We have developed an R package to conduct a parent-offspring test of individuals which are genotyped with a fixed set of SNP markers for further genetic studies. The program uses monomorphic SNP loci between parents and their progeny genotypes to calculate the similarity between each offspring and their parents. Based on the similarity of parents and individual offspring, the users can determine the threshold level for the individuals to be included for further data analysis. We used an $F_{5}$-derived soybean population of '5601T' x PI 157440 that was genotyped with 1,536 SNPs to illustrate the procedure and its application.

Conclusions: The R package 'ParentOffspring' coupled with the available SNP genotyping platforms could be used to detect the possible variants in a specific cross, as well as the potential errors in sample handling and genotyping processes. It can be used in any crop which is genotyped with a fixed set of SNP markers.
\end{abstract}

Keywords: SNP markers, Genotyping, Parent-offspring tests

\section{Background}

Single nucleotide polymorphism (SNP) genotyping platforms including Invader ${ }^{\circ}$ assay, single base extension (SBE), oligonucleotide ligation assay (OLA) SNPlex ${ }^{\mathrm{Th}}$ system, and the Illumina GoldenGate ${ }^{\mathrm{Tm}}$ and Infinium ${ }^{\mathrm{Tw}}$ assays [1] have been developed and widely used to genotype crop plants with a fixed set of SNP markers [2-8]. The SNP data generated from these platforms have been extensively used for genetic and genomic studies including QTL mapping, germplasm characterization, association mapping, and molecular breeding. With a large scale of SNP data available for data analysis, integrity of individuals included in an experiment is very important to ensure the accuracy of the results. For example in a typical QTL mapping population or progeny derived from a cross, variants could result from outcrossing, seed mixing, sampling errors, and many other ways during the

\footnotetext{
*Correspondence: zli@uga.edu

'Institute of Plant Breeding, Genetics and Genomics \& Center for Applied Genetic Technologies, University of Georgia, 111 Riverbend Rd., Athens, GA 30602, USA

Full list of author information is available at the end of the article
}

population development and sample handling processes. When variants are present, they could distort the experimental results. Although morphological and physiological characteristics can be used to distinguish the variants in a population or seed lot, they are very limited due to availability of phenotypes and accuracy of determination. With the availability of a high density of SNP markers assayed on these advanced genotyping platforms, monomorphic marker loci could be used to detect outcrossing or seed mixture of individuals and possible genotyping errors during sampling and genotyping processes by comparing the SNP alleles of these progeny with their parents' alleles. In soybean, the Universal Soy Linkage Panel (USLP 1.0) consisting of 1,536 SNPs on the Illumina GoldenGate ${ }^{\bullet}$ Platform has been developed and used for quantitative trait locus (QTL) discovery [6]. Recently, the iSelect Infinium assay which contained over 50,000 SNPs from soybean genome have been also developed [9]. Yan et al. (2009) genotyped 632 inbred maize lines with 1,536 SNPs. Over

Ciomed Central

(c) 2013 Abdel-Haleem et al.; licensee BioMed Central Ltd. This is an Open Access article distributed under the terms of the Creative Commons Attribution License (http://creativecommons.org/licenses/by/2.0), which permits unrestricted use, distribution, and reproduction in any medium, provided the original work is properly cited. The Creative Commons Public Domain Dedication waiver (http://creativecommons.org/publicdomain/zero/1.0/) applies to the data made available in this article, unless otherwise stated. 
Table 1 An example of assigning numerical $(0,1,2)$ scores based on the genotypes of progeny and their parents ( $x$ represents an SNP allele from both parent 1 and parent 2 and $y$ indicates that the SNP allele is not from parent 1 or parent 2)

\begin{tabular}{llll}
\hline Parent 1 & Parent 2 & Progeny & Score \\
\hline$x x$ & $x x$ & $x x$ & 2 \\
$x x$ & $x x$ & $x y$ & 1 \\
$x x$ & $x x$ & $y y$ & 0 \\
\hline
\end{tabular}

200 barley germplasm lines, including European and U.S. breeding materials, were genotyped with 3,072 SNPs on Illumina GoldenGate assays that are available to the barley community [2]. Similarly, 1,536 SNPs on Illumina GoldenGate assays were developed to fingerprint 478 spring and winter wheat lines [8]. A custom-designed Affymetrix array consisting of 44,100 SNPs was used in rice to study the genetic architecture of aluminum tolerance in a biparental population and a set of 383 diverse rice accessions $[10,11]$.

With such advanced genotyping technologies, massive amount of SNP data including both polymorphic and monomorphic loci have been generated. Typically, the monomorphic loci are excluded from the data set before further analyses. For example, genotyping a bi-parental population in soybean using an USLP 1.0 panel of 1,536 SNP loci will result in around 1,000 monomorphic loci, which could be used to test if all progeny are truly derived from same parents. Here we developed an R package to calculate the similarity between each offspring and its parents using monomorphic SNP loci. This program can be used by researchers to quality control the

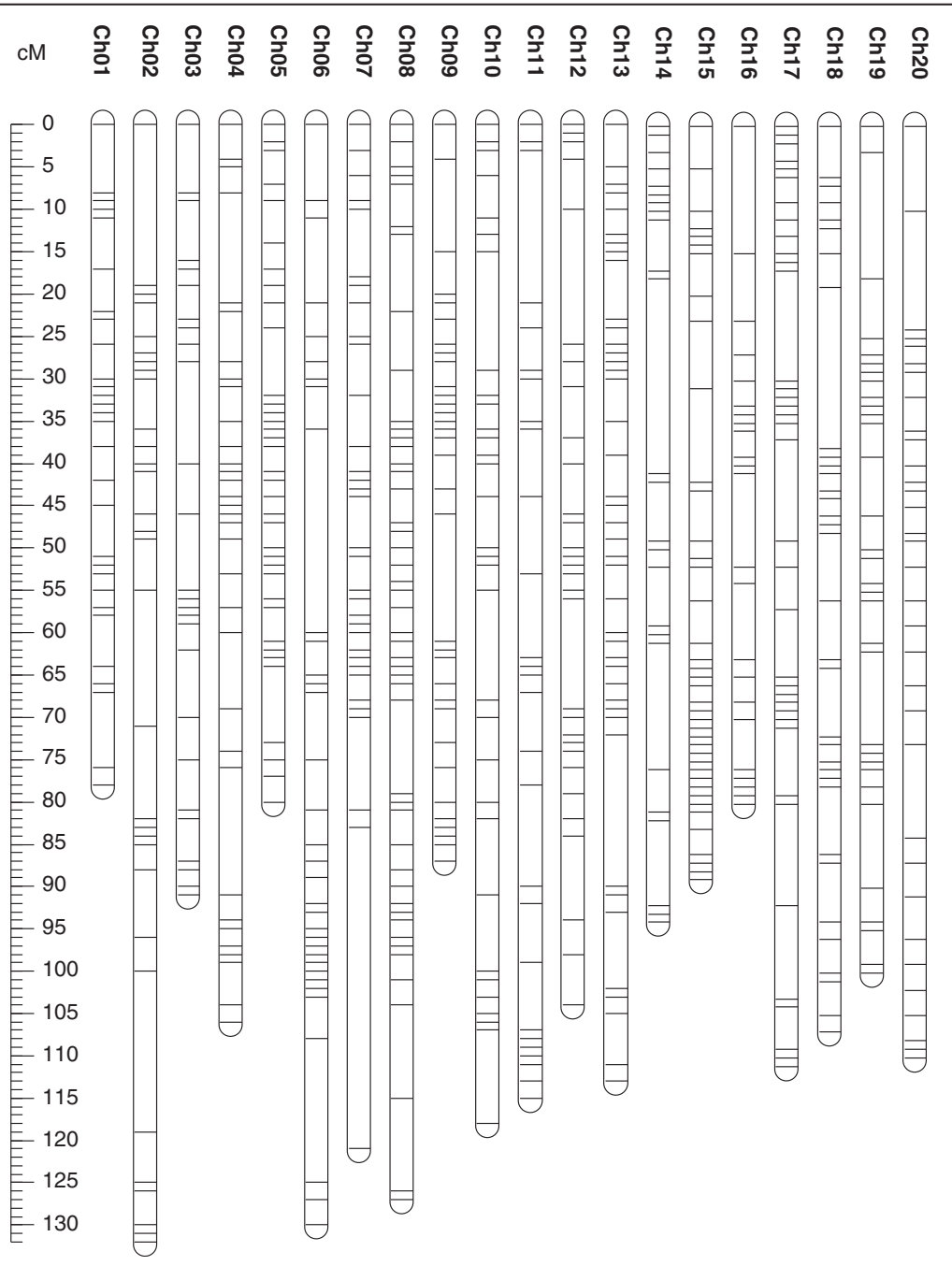

Figure 1 Distribution of monomorphic SNP markers on 20 soybean chromosomes generated from 5601T x PI 157440 population. 


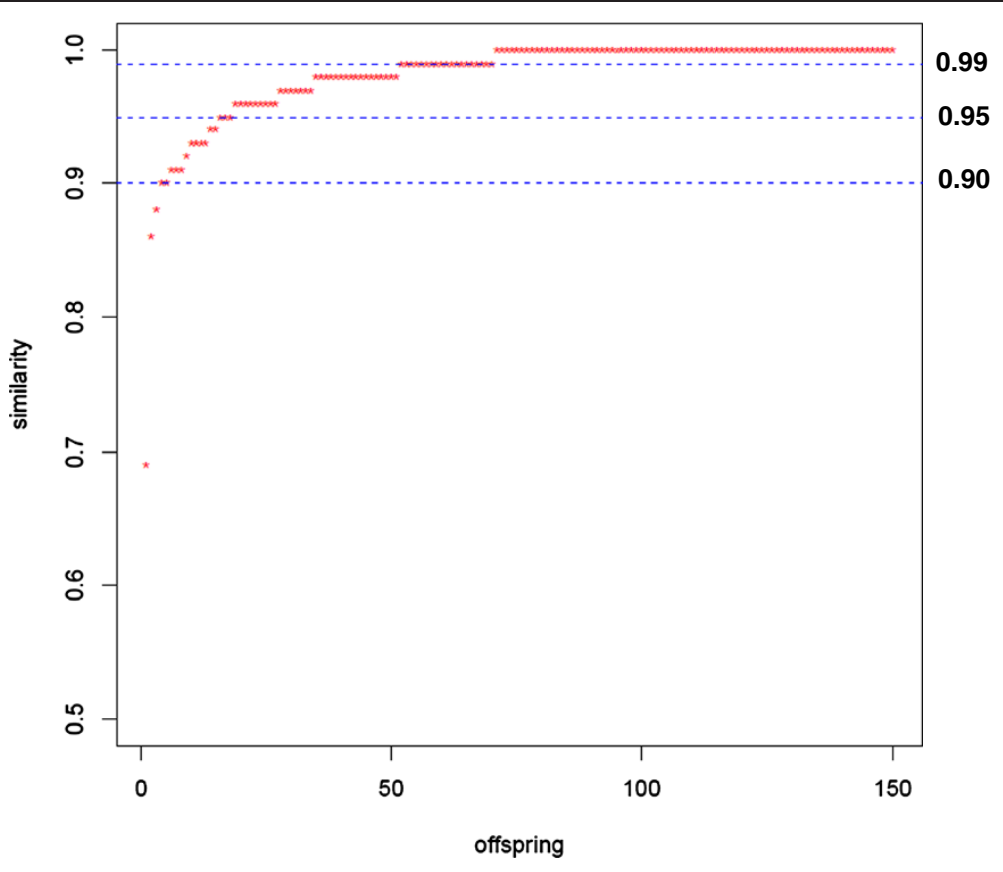

Figure 2 Distribution of parent-offspring similarity values.

progeny and determine which offspring needs to be excluded from further analysis.

\section{Implementation}

To calculate the similarity of each offspring to its parents utilizing the parental monomorphic loci at each monomorphic SNP locus, allele calls in each offspring are assigned with numbers 0,1 , and 2 by comparing the parental genotype. A score of " 2 " is assigned when an offspring has same alleles to the both parents; a score of "1" indicates that the offspring possesses one parental allele and one non-parental allele; and a score of "0" indicates that the offspring possesses different alleles than their parents. An example of the assignments is presented in Table 1.

The similarity between an offspring and its parents is calculated based on the all monomorphic loci as follow:

$$
\mathrm{S}=(2 \mathrm{a}+\mathrm{b}) /(2 \mathrm{a}+2 \mathrm{~b}+2 \mathrm{c})
$$

Where $\mathrm{S}=$ the similarity between an offspring and its parents, $a=$ the number of markers with a score of 2 , and $b=$ the number of markers with a score of 1 and $c=$ the number of markers with a score of 0 . The $\mathrm{R}$ package

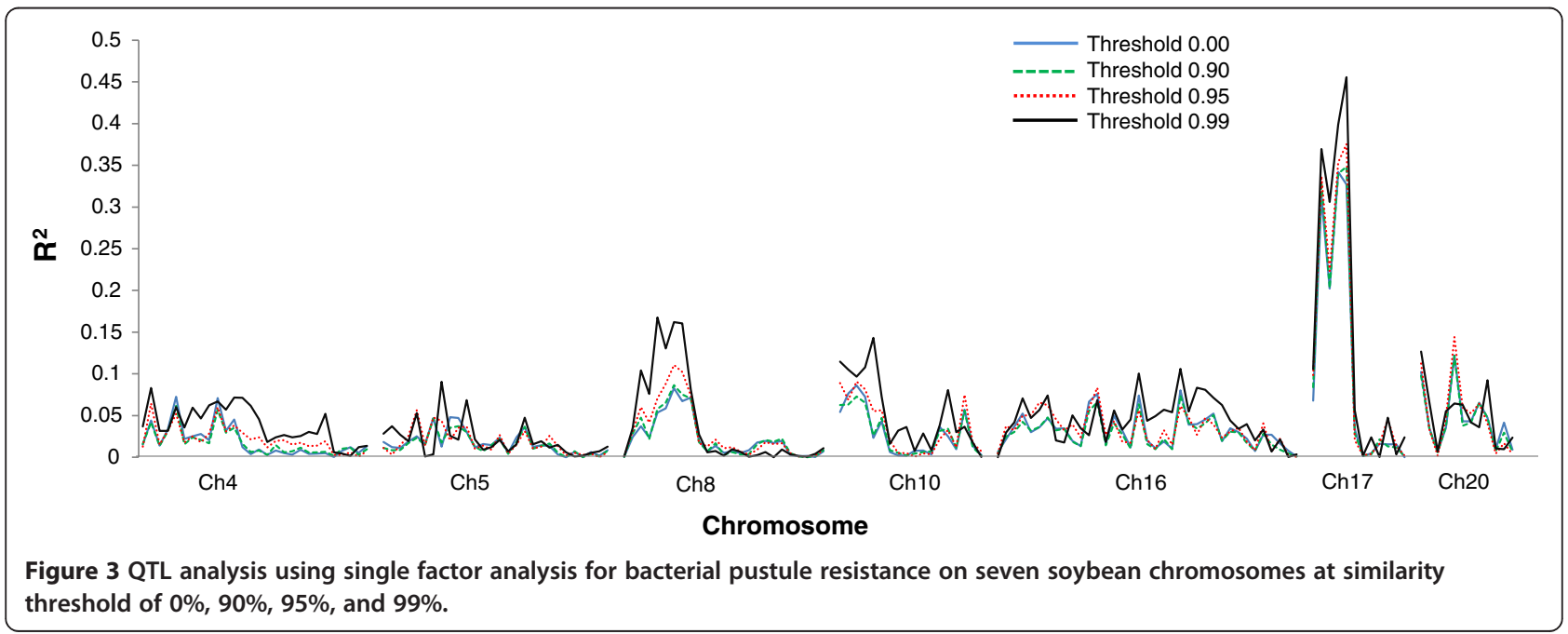


can be found in following link (http://cran.r-project.org/ web/packages/ParentOffspring/index.html). The output files of this program include both monomorphic and polymorphic SNP marker data set for further analyses.

Theoretically, an offspring should have 100\% similarity to its parents. However, due to the possible genotyping errors, outcrossing, seed mixture, and other unknown reasons, similarity of an offspring to its parents could be less than $100 \%$. Reduced similarity will increase the possibility of that offspring being a variant of the specific cross.

\section{Result and discussion}

An $\mathrm{F}_{5}$-derived recombinant inbred line soybean population was used as an example to illustrate the procedure and application. The population was developed from a cross of $5601 \mathrm{~T}$ x PI 157440 at the University of Georgia Plant Sciences Farm, Athens, Georgia. 5601T is a cultivar developed and released by University of Tennessee [12] and PI 157440 was selected as a parent based on its high canopy photosynthetic capacity during the reproductive period [13]. The $F_{1}$ plants were selfed to produce $F_{2}$ plants. Seeds from individual $F_{2}$ plants were advanced to the $F_{5}$ generation using a single-seed descent method [14]. The $\mathrm{F}_{5}$ plants were grown at the University of Georgia Plant Sciences Farm and at maturity, individual $\mathrm{F}_{5}$ plants were harvested to create $150 \mathrm{~F}_{5: 6}$ lines. The soybean USLP 1.0 panel of 1,536 SNP markers on the GoldenGate ${ }^{\oplus}$ platform [6] were used to fingerprint these 150 RILs and their parents. The SNP allele calls were performed on the Illumina BeadStation 500G (Illumina, San Diego, CA). The population was evaluated to the bacterial pustule disease reaction (Xanthomonas campestris pv. Glycines) under field conditions of UGA Plant Sciences Farm during 2010 in a randomized complete block design with two replications. Based on leaf severity symptoms, the lines were visually rated for bacterial pustule reaction on a plot basis with a scale of 1 to 5 , where plots with no symptoms were rated as 1 (Resistant) and plots with severe symptoms as 5 (Susceptible). Associations of the SNP markers with the bacterial pustule rates were tested using single-factor analysis in SAS 9.3 [15].

Of 1,536 SNP markers in $5601 \mathrm{~T} \times$ PI 157440 population, 542 SNPs (37\%) were polymorphic and 938 SNPs monomorphic which accounted for $63 \%$ of the total SNP markers. These monomorphic SNPs were distributed eventually on all chromosomes (Figure 1).

When the similarity threshold was set as $90 \%$, five genotypes were declared as variants; at similarity ratio $\leq$ $95 \%$, the number of variants was 18 , and at more stringent similarity ratio of $\leq 99 \%$, the number of variants reached 70 (Figure 2). The determination of similarity threshold level depends on many factors such as objectives, line types, and genotyping platforms. It is expected that excluding the variants from a population will lead to eliminate the outliners for further genetic analysis. To demonstrate the method, the polymorphic SNP markers data from the 150 RILs of $5601 \mathrm{~T} \times$ PI 157440 population were used to detect QTL associated with resistance to bacterial pustule disease using a single factor analysis approach. Single factor analysis identified a major QTL accounted for 32.7\% of the phenotypic variation on chromosome 17 that is in agreement with the reports by Narvel et al. [16]. Based on the similarity threshold levels of 90, 95, and 99\% (Figure 3), 5, 18, and 70 lines, respectively, were excluded from the dataset for analysis. The $\mathrm{R}^{2}$ for the major QTL on chromosome 17 becomes $34.8,37.5$ and $45.6 \%$, respectively (Figure 3 ). This indicated that quality control of the offspring using the monomorphic SNP markers could help improving the genetic analyses and thus accuracy of the result. Based on our data, we suggest to use the similarity ratio of $90-95 \%$ as a threshold in a study.

\section{Conclusions}

The R package 'ParentOffspring' was developed to conduct a parent-offspring test of individuals which are genotyped with a fixed set of SNP markers for further genetic studies. The application of the $\mathrm{R}$ package coupled with the available SNP genotyping platforms could be used to detect the possible variants in a specific cross, as well as the potential errors in sample handling and genotyping processes. It can be used in any crop which is genotyped with a fixed set of SNP markers.

\section{Availability and requirements}

Project name: ParentOffspring project

Project home page: http://cran.r-project.org/web/packages/ParentOffspring/

Operating system(s): Windows, Mac OS, Linux

Programming language: $\mathrm{R}$

Other requirements: $\mathrm{R}$ version 2.15.1 or higher

License: GPL-2 | GPL-3

Any restrictions to use by non-academics: Non.

\section{Abbreviations}

QTL: Quantitative trait loci; SNP: Single nucleotide polymorphism.

\section{Competing interests}

The authors declare that they have no competing interests.

\section{Authors' contributions}

HA generated the experimental data, ran the R package and wrote the MS. $H A, P J$ and $Z \mathrm{~L}$ developed the procedure, implemented the R package, and evaluated the program. HRB generated the mapping population. All authors participated in the design of the study, edited and approved the final manuscript.

\section{Acknowledgement}

This research was supported by funds allocated to the Georgia Agricultural Experiment Stations and funding from the United Soybean Board. 


\section{Author details}

'Institute of Plant Breeding, Genetics and Genomics \& Center for Applied Genetic Technologies, University of Georgia, 111 Riverbend Rd., Athens, GA 30602, USA. ${ }^{2}$ Department of statistics, University of Georgia, 103 Statistics Building, 101 Cedar St., Athens, GA 30602, USA. ${ }^{3}$ Georgia Seed Development Commission, 2420 S. Milledge Av., Athens, GA 30605, USA.

Received: 8 October 2013 Accepted: 5 November 2013

Published: 19 November 2013

\section{References}

1. Appleby N, Edwards D, Batley J: New technologies for ultra-high throughput genotyping in plants. Methods Mol Biol 2009, 513:19-39.

2. Close T, Bhat P, Lonardi S, Wu Y, Rostoks N, Ramsay L, Druka A, Stein N, Svensson J, Wanamaker $S$, et al: Development and implementation of high-throughput SNP genotyping in barley. BMC Genomics 2009, 10(1):582.

3. Yan JB, Yang XH, Shah T, Sanchez-Villeda H, Li JS, Warburton M, Zhou Y, Crouch JH, Xu YB: High-throughput SNP genotyping with the GoldenGate assay in maize. Mol Breed 2009, 25:441-451.

4. Deulvot C, Charrel H, Marty A, Jacquin F, Donnadieu C, Lejeune-Henaut I, Burstin J, Aubert G: Highly-multiplexed SNP genotyping for genetic mapping and germplasm diversity studies in pea. BMC Genomics 2010, 11(1):468.

5. McCouch S, Tung C-W, Zhao K, Wright M, Kimball J, Reynolds A, Tyagi W, Wang D, Declerck G, Ali ML, et al: Development of genome-wide SNP assays for rice. Breeding Sci 2010, 60:524-535.

6. Hyten DL, Choi IY, Song Q, Specht JE, Carter TE, Shoemaker RC, Hwang EY, Matukumalli LK, Cregan PB: A high density integrated genetic linkage map of soybean and the development of a 1536 universal soy linkage panel for quantitative trait locus mapping. Crop Sci 2010, 50(3):960-968.

7. Bachlava E, Taylor CA, Tang S, Bowers JE, Mandel JR, Burke JM, Knapp SJ: SNP discovery and development of a high-density genotyping array for sunflower. Plos One 2012, 7(1):e29814.

8. Chao S, Dubcovsky J, Dvorak J, Luo M-C, Baenziger S, Matnyazov R, Clark D, Talbert L, Anderson J, Dreisigacker S: Population- and genome-specific patterns of linkage disequilibrium and SNP variation in spring and winter wheat (Triticum aestivum L.). BMC Genomics 2010, 11(1):727.

9. Song Q, Hyten DL, Jia G, Quigley CV, Fickus EW, Nelson RL, Cregan PB: Development and evaluation of SoySNP50K, a high-density genotyping array for soybean. Plos One 2013, 8(1):e54985.

10. Famoso AN, Zhao K, Clark RT, Tung C-W, Wright MH, Bustamante C, Kochian LV, McCouch SR: Genetic architecture of aluminum tolerance in rice (Oryza sativa) determined through genome-wide association analysis and QTL mapping. PLoS Genet 2011, 7(8):e1002221.

11. Tung C-W, Zhao K, Wright M, Ali ML, Jung J, Kimball J, Tyagi W, Thomson M, McNally K, Leung H: Development of a research platform for dissecting phenotype-genotype associations in rice (Oryza spp.). Rice 2010, 3(4):205-217.

12. Pantalone VR, Allen FL, LandauEllis D: Registration of '5601T' soybean. Crop Sci 2003, 43(3):1123-1124

13. Boerma H, Ashley D: Canopy photosynthesis and seed-fill duration in recently developed soybean cultivars and selected plant introductions. Crop Sci 1988, 28(1):137-140.

14. Brim C: A modified pedigree system of selection in soybeans. Crop SC 1966, 6:220.

15. SAS: SAS user's quide Version 9.3. Cary, NC: SAS Institute Inc; 2011.

16. Narvel J, Jakkula L, Phillips D, Wang T, Lee SH, Boerma H: Molecular mapping of Rxp conditioning reaction to bacterial pustule in soybean. J Heredity 2001, 92(3):267-270.

doi:10.1186/1746-4811-9-44

Cite this article as: Abdel-Haleem et al:: An R package for SNP marker-

based parent-offspring tests. Plant Methods 2013 9:44.

\section{Submit your next manuscript to BioMed Central and take full advantage of:}

- Convenient online submission

- Thorough peer review

- No space constraints or color figure charges

- Immediate publication on acceptance

- Inclusion in PubMed, CAS, Scopus and Google Scholar

- Research which is freely available for redistribution 